\title{
USE AND ABUSE OF JUNGMANN'S METHOD FOR THE LIQUEFACTION OF TUBERCULOUS SPUTA
}

\author{
BY \\ K. ANDERSON, J. A. HUGHES, AND R. KNOX \\ From the Department of Bacteriology, Guy's Hospital Medical School, London
}

(RECEIVED FOR PUBLICATION FEBRUARY 19, 1953)

Of the many chemical methods designed to aid the demonstration of acid-fast bacilli in sputum, the majority prove unsatisfactory in some respect when tested in the laboratory. The ideal method must first kill contaminating organisms in the specimen and liquefy or homogenize the mucopurulent constituents so that bacilli present can be concentrated by centrifugation. It must also leave deposited bacilli which are viable and which can still be recognized when stained by standard methods. Griffith (1914) first used antiformin to destroy contaminating organisms. Petroff (1915) employed $3 \% \mathrm{NaOH}$, while Corper and Uyei (1927) found $6 \% \mathrm{H}_{2} \mathrm{SO}_{4}$ superior to $\mathrm{NaOH}$. In a preliminary paper Jungmann (1938) described an iron-acid peroxide method in which varying concentrations of reagents were tried for the liquefaction of tuberculous sputa. In a later paper Jungmann and Gruschka (1938) stated the concentrations of solutions which had proved most efficient. These are as follows:

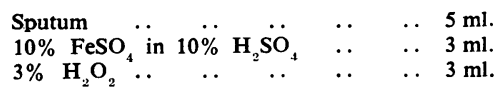

The mixture is allowed to react for five minutes with stirring, centrifuged for five minutes, and the deposit neutralized with either $2 \%$ sodium lactate, $2 \%$ sodium citrate, or several washes with normal saline.

Since the publication of Jungmann's method, a number of minor modifications have been made in the technique. These mainly affect the time taken for liquefaction and the duration of centrifugation. However, several more radical alterations have appeared in the literature over the intervening years. These affect the volume strength of hydrogen peroxide used in digestion and the treatment of the centrifuged deposit when digestion is complete. In a survey of sputum concentration methods Baker (1951) concludes that Jungmann's technique is inferior to four other methods, both for films and culture. A working party of the
Public Health Laboratory Service (Ministry of Health . Report, 1952) has recently reported on a large-scale survey of methods of homogenization. For all classes of specimen, the order of success places Jungmann's third on the list of four methods examined.

During a trial of isoniazid in the treatment of pulmonary tuberculosis (Joiner, MacLean, Pritchard, Anderson, Collard, King, and Knox, 1952) it was decided to adopt Jungmann's technique for examining sputa before the contents of the working party's report were available. We used the method as originally described by Jungmann and Gruschka (1938), and, in referring to this, observed that the method attributed to Jungmann in the Report and also by Baker (1951) and Collins (1952) contained several departures from the original. It was therefore decided to investigate what effect such modifications might have on the efficiency of the method. These alterations in technique are now considered in detail.

\section{Volume Strength of Hydrogen Peroxide}

Jungmann and Gruschka state that $3 \%$ hydrogen peroxide is the most effective concentration for routine use. Since a $1 \%$ solution $(w / v)$ will give $-\frac{22.4}{68} \times 10=3.3$ litres of oxygen per litre of solution at N.T.P., a $1 \%$ solution is equivalent to a volume strength of 3.3. Thus, for all practical purposes, a $3 \%$ solution is identical to 10 volume hydrogen peroxide normally dispensed. The expression "volume per cent," which is frequently quoted in descriptions of this method, must be considered meaningless, and any reference to solutions of hydrogen peroxide should be made either in terms of volume strength or percentage. An alteration in the volume strength of hydrogen peroxide has appeared in all the accounts of Jungmann's method studied during this investigation. Nassau (1942) describes the use of " 1 volume \%," which is taken to mean a 1 -volume solution. In 
the proportions used, this gives a final concentration of only 0.3 volume hydrogen peroxide $(0.1 \%)$ in the mixture with sputum added compared with 2.7 volume $(0.8-0.9 \%)$ when a 10 volume solution is used. In a report on the demonstration of tubercle bacilli in infected material in the Monthly Bulletin of the Ministry of Health (1945) the use of a 1-volume solution is again described, and this is repeated in the paper by Baker (1951) and in that by Collins (1952). The Report describes the use of slightly different amounts of sputum and reacting solutions, but the final concentration of hydrogen peroxide in the mixture using a 1-volume solution remains at 0.3 volume $(0.1 \%)$. Reference to standard textbooks shows that the use of weaker solutions is still recommended and attributed to Jungmann. Recent Advances in Clinical Pathology (Dyke, 1951) and Medical Bacteriology (Whitby and Hynes, 1951) both describe the use of 1-volume solutions in the standard technique.

It is clear, therefore, that there exists a widespread but erroneous practice which involves the use of peroxide solutions of approximately $1 / 10$ of the concentration originally recommended by Jungmann and Gruschka. We therefore investigated the effect of varying volume strengths of hydrogen peroxide upon the speed and efficacy of liquefaction of sputum samples.

Experimental.-Specimens of mucopurulent sputa were divided into approximately $5-\mathrm{ml}$. amounts in four separate $15-\mathrm{ml}$. conical centrifuge tubes. Care was taken that there was an equal division of purulent and mucoid elements so that the tubes should be as nearly comparable as possible. Then $3 \mathrm{ml}$. of $10 \% \mathrm{FeSO}_{4}$ in $10 \% \mathrm{H}_{2} \mathrm{SO}_{4}$ was added to each tube followed by $3 \mathrm{ml}$. of one of the following volume strengths of hydrogen peroxide: $10,5,2$, and 1 . The tubes were stoppered and allowed to stand at room temperature for 15 minutes, shaking at five-minute intervals. At the end of the first five minutes it was clear that complete liquefaction had occurred in the tubes containing 10- and 5-volume solutions. Both tubes showed a thin, homogeneous fluid in which fine particles were evenly distributed. The remaining tubes containing 2- and 1-volume hydrogen peroxide still showed masses of stringy mucus which floated to the surface after shaking. This fully confirmed the original observation of Jungmann and Gruschka that five minutes was an adequate time for liquefaction when 10-volume solutions were used. However, all tubes were allowed to stand for a further 10 minutes in case the weaker solutions could effect liquefaction in this time. They were then centrifuged at 3,000 r.p.m. for 15 minutes and examined. Fig. 1 shows the appearance of the tubes after centrifugation. The incomplete liquefaction produced by the lower volume strengths is clearly seen, and is in striking contrast

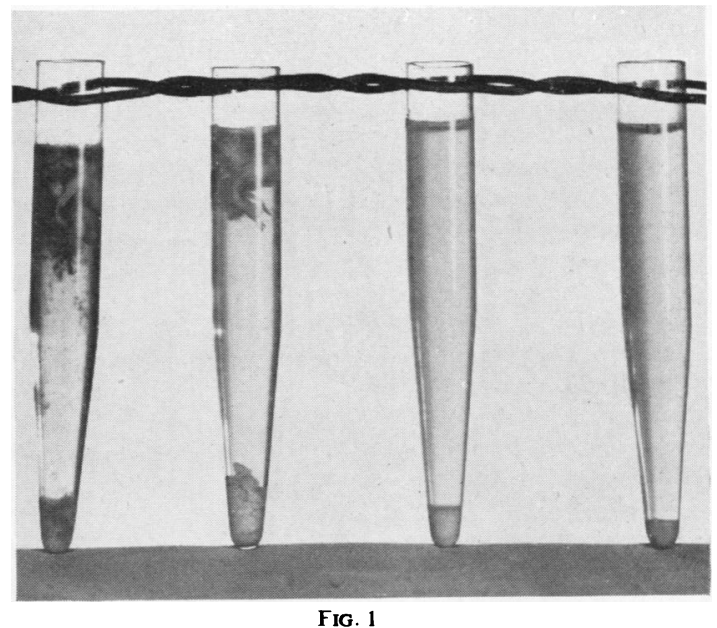

with the compact deposit obtained with 10-volume solutions. Although a more complete degree of liquefaction was occasionally obtained when watery specimens of sputum were treated, most mucopurulent sputa, so characteristic of cavitating pulmonary tuberculosis, were incompletely digested when 1-volume solutions were used.

\section{Neutralization of Centrifuged Deposits}

Jungmann and Gruschka state that deposits should be neutralized with 10 to $15 \mathrm{ml}$. of either $2 \%$ sterile sodium lactate or $2 \%$ sodium citrate, followed by re-centrifugation. Alternatively, two separate washes with normal saline may be employed. However, Recent Advances in Clinical Pathology (1942) gives neutralization with $\mathrm{N} / \mathrm{I} \mathrm{NaOH}$ as an alternative which avoids recentrifugation of the deposit. Baker (1952) also used this method in his survey. Preliminary experiments have shown this to be an exceedingly diffcult procedure.

Experimental.-If $\mathrm{N} / \mathrm{I} \mathrm{NaOH}$ is added dropwise to a deposit in the presence of an indicator such as phenol red, phenolphthalein, or " universal " (B.D.H.) a dense precipitate of ferric hydroxide is formed from the interaction of alkali and residual ferrous sulphate. This precipitate appears before the indicator gives any warning of approaching neutrality. One such " neutralized" deposit from a strongly positive sputum failed to yield any colonies after six weeks' culture on Löwenstein-Jensen medium. An attempt was then made to neutralize small quantities of iron-acid peroxide mixture alone in the test-tube, bearing in mind the possibility that deposited sputum might obscure slight changes in the indicator. It was not possible, using either indicator, to detect an alteration towards neutrality before ferric hydroxide was precipitated. When weaker solutions of $\mathrm{NaOH}$ were used more accurate neutralization was possible, but the volumes 
involved were large. Re-centrifugation would therefore have been necessary when dealing with sputum, and in these circumstances the use of lactate or saline is both simpler and less time-consuming. It may be added that neutralization of a deposit by re-suspension in a large volume of fluid is to be preferred when dealing with specimens from treated cases, since antituberculous drugs which may be excreted in the sputum are more likely to be washed from the deposit and discarded in the supernatant fluid.

It seems, therefore, that the use of $\mathrm{N} / \mathrm{I} \mathrm{NaOH}$ as a neutralizing agent is a difficult and perhaps lethal procedure. Apart from the problem of detecting changes in the indicator and the danger of adding excess alkali, it is likely that precipitated ferric hydroxide is toxic to the tubercle bacillus in this concentration.

\section{Microscopy of Centrifuged Deposits}

The value of Jungmann's method in the preparation of smears from digested sputum is probably not widely recognized. It is of interest to quote the author's original remarks on microscopical examination :

"The sediment is easily, cleanly, and evenly smeared on a slide and adheres to the glass. Staining by the $\mathrm{Z}-\mathrm{N}$ technique gives clear pictures that are free from artefacts, but it must be noted that the staining is inhibited by traces of residual acid, and the above washings must be carefully carried out. Cells appear slightly pyknotic, but the tubercle bacillus stains brightly and stands out well against a short application of methylene blue."

These findings have been fully confirmed in our experience. It is our impression that the bacilli do, in fact, show a more vivid pillar-box red after digestion and washing, and are readily seen against a counterstain of malachite green or methylene blue.

The question arises as to the advisability of preparing smears from unwashed deposits. Baker used this method in his survey before staining by the auramine-phenol technique. It was decided to test the effect of residual acid on the staining properties of smears in view of the warning given by Jungmann and Gruschka.

Experimental.-Smears from a known positive sputum were prepared and stained by the ZiehlNeelsen technique. The specimen was then concentrated by Jungmann's method, and further smears made from the unwashed and the washed deposit. These were also stained by the Ziehl-Neelsen method. It was at once noted that the unwashed smears did not dry with normal speed at room temperature. This was presumably due to the hygroscopic nature of the residual acid. Any attempt to fix the smear or hasten drying by gentle heat resulted in immediate charring of the deposit. When such a smear was stained the cellular background was found to be completely destroyed and no recognizable bacilli could be seen. Other preparations which were not fixed showed a moderately well preserved cellular deposit, but the tubercle bacilli did not stain satisfactorily. Although some appeared red, others, recognizable only by their morphology, stained a blue-black and had obviously been partially destroyed. The washed deposit, however, appeared satisfactory in every respect, and showed adequate concentration of bacilli when compared with the original smear.

Jungmann's statement appears fully justified, since residual acid affects both the cellular elements and the staining properties of the tubercle bacillus. The use of even gentle heat for fixation results in complete destruction of the smear.

\section{Discussion}

The alterations in Jungmann's method which have appeared in the literature since its original description can clearly detract from its efficiency in several ways. The sputum may be inadequately digested and concentrated, the viability of the bacilli endangered by incorrect neutralization, and the staining properties impaired by the use of acid deposits. However, the full significance of these modifications cannot be assessed without a largescale trial of the type recently reported by the Public Health Laboratory Service. A search of the literature fails to reveal any survey of methods in which Jungmann's technique is on trial in its original form. While we in no way desire to criticize the factual material presented by the various workers in this field, it is clear that the iron-acid peroxide method has been placed in an inferior position vis $\grave{a}$ vis homogenization techniques, which it does not justly deserve.

In this laboratory the scantily positive specimen has not been available in sufficiently large numbers to justify a critical trial. However, during the recent clinical trial of isoniazid (Joiner et al., 1952) the results of culture of some 426 specimens concentrated by Jungmann's method gave no cause for dissatisfaction. For example, of 172 control sputa, N $162(94 \%)$ were positive on culture. Details of $N$ these specimens are as follows:

$$
\begin{array}{llllc}
\text { Z-N + ve Culture +ve } & \ldots & \ldots & \ldots & 138(80 \%) \\
\text { Z-N - ve Culture +ve } & \ldots & \ldots & \ldots & 24(14 \%) \\
\text { Z-N + ve Culture - ve } & \ldots & \ldots & \ldots & 0 \\
\text { Z-N - ve Culture - ve } & \ldots & \ldots & \ldots & 10(6 \%)
\end{array}
$$

Of 521 tubes inoculated, $22(4 \%)$ were contam- $\stackrel{\mathscr{D}}{\rightleftharpoons}$ inated. This compares favourably with the sodium 0 hydroxide method as quoted by the Public Health ${ }_{0}$

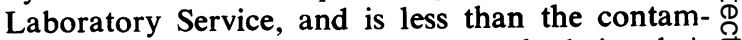
ination rate with Jungmann's method in their $\frac{\overrightarrow{\mathbb{D}}}{\mathbb{Q}}$ hands $(5.2 \%)$.

In a small pilot survey by one of us Jungmann's method has been compared with $4 \% \mathrm{NaOH}$. A 8 
hundred specimens were selected, of which 61 were negative on direct microscopy. All the specimens were obtained from patients under treatment with a variety of anti-tuberculosis drugs. The results are analysed in Tables I and II.

TABLE I

COMPARISON OF JUNGMANN AND SODIUM HYDROXIDE METHODS IN 100 SPECIMENS OF SPUTUM

\begin{tabular}{|c|c|c|c|c|}
\hline & $\begin{array}{c}\text { Jungmann } \\
\text { Positive } \\
\text { Soda } \\
\text { Positive }\end{array}$ & \begin{tabular}{|} 
Jungmann \\
Positive \\
Soda \\
Negative
\end{tabular} & $\begin{array}{l}\text { Jungmann } \\
\text { Negative } \\
\text { Soda } \\
\text { Positive }\end{array}$ & \begin{tabular}{|c} 
Jungmann \\
Negative \\
Soda \\
Negative
\end{tabular} \\
\hline $\begin{array}{l}\text { Total specimens cul- } \\
\text { tured, } 100 \\
\text { Specimens Ziehl- } \\
\text { Neelsen negative, } \\
61 \\
\text { Specimens Z Ziehl- } \\
\text { Neelsen positive, } \\
39\end{array}$ & $\begin{array}{c}3(5 \%) \\
15(38 \%)\end{array}$ & $\begin{array}{l}4(6 \%) \\
9(23 \%)\end{array}$ & $\begin{array}{l}6(10 \%) \\
5(12 \%)\end{array}$ & $\begin{array}{c}58 \% \\
48(79 \%) \\
10(27 \%)\end{array}$ \\
\hline
\end{tabular}

TABLE II

APPEARANCE OF FIRST POSITIVE CULTURE (AVERAGE NUMBER OF WEEKS)

\begin{tabular}{|c|c|c|c|c|}
\hline \multirow{2}{*}{ Method } & \multicolumn{4}{|c|}{ Microscopy } \\
\hline & Negative & Scanty & Moderate & Heavy \\
\hline $\begin{array}{l}\text { Jungmann } \\
\text { Soda }\end{array}$ & $\begin{array}{l}4 \cdot 5 \\
5 \cdot 3\end{array}$ & $\begin{array}{l}4.5 \\
5.5\end{array}$ & $\begin{array}{l}4 \cdot 0 \\
4 \cdot 2\end{array}$ & $\begin{array}{l}4 \cdot 5 \\
4 \cdot 1\end{array}$ \\
\hline
\end{tabular}

These results support our belief that Jungmann's original method is at least not inferior to the alkali method of digestion. All those who have worked with the technique in this laboratory have found sputa easier and cleaner to handle than with other methods, and therefore a wider margin of safety is provided when dealing with larger numbers of specimens. We feel that the technique deserves further extensive laboratory trials before its exact place amongst other methods can be accurately determined.

\section{Summary}

Attention is drawn to several departures from Jungmann's original method which have appeared in the literature over a number of years.

These may materially affect the efficiency of the method by resulting in poor concentration, reduced viability of bacilli, or diminished staining properties.

Results obtained with Jungmann's original method are analysed and a plea is made for further extensive laboratory trials to establish its position amongst other techniques of digestion.

We are indebted to the Photographic Department, Guy's Hospital, for Fig. 1. Our thanks are also due to Miss U. Offenbacher for her help in translating Jungmann's papers.

\section{REFERENCES}

Baker, F. J. (1951). J. med. Lab. Technol., 9, 160.

Collins, C. H. (1952). Tubercle, 33, 149.

Corper, H. J., and Uyei, N. (1927). Amer. Rev. Tuberc., 16, 299.

Dyke, S. C., ed. (1951). Recent Advances in Clinical Pathology, 2nd

ed. Churchill, London.

Joiner, C. L., MacLean, K. S., Pritchard, E. K., Anderson, K. Collard, P., King, M. B., and Knox, R. (1952).' Lancet, 2, 843.

Jungmann, K. (1938). Klin. Wschr., 17, 238.

and Gruschka, T. (1938). Ibid., 17, 239.

Ministry of Health Report (1945). London Sector Pathologists, Monthly Bull. Minist. Hlth, Lond., 4, 202.

(1952). Report by a Working Party. Public Health Laboratory Service. Ibid., 11, 187.

Nassau, E. (1942). Tubercle, 23, 179

Petroff, S. A.. (1915). J. exp. med., 21, 38

Whitby, L., and Hynes, M. (1951). Medical Bacteriology, 5th ed. Churchill, London. 